\title{
Long-term administration of Active Hexose Correlated Compound as a dietary supplement to a patient after breast cancer surgery and chemotherapy: A case report
}

\author{
Koji Wakame $^{1 *}$, Jun Takanari ${ }^{2}$, Atsuya Sato ${ }^{2}$, Satomi Shirakawa ${ }^{2}$ and Ken-ichi Komatsu ${ }^{1}$ \\ ${ }^{1}$ Department of Pharmacology, Hokkaido Pharmaceutical University School of Pharmacy, Japan \\ ${ }^{2}$ Science and Development Group, Amino Up Chemical Co. Ltd. , Japan
}

\begin{abstract}
Active Hexose Correlated Compound (AHCC) is a dietary supplement obtained by long-term liquid culturing of shiitake mycelia. AHCC has various biological functions; in particular, its immune stimulating effect is well known. In recent years, the use of patients for treatment of cancer is increasing in the field of integrated medical care under the initiative of doctors and other paramedic experts. Practically, AHCC research from both animal models and human clinical trials has been reported, but an insufficient number of individual cases have been reported. Therefore, we report the case of a breast cancer patient who took AHCC ( 3 g/day) for 9 years following breast cancer surgery and chemotherapy treatment.
\end{abstract}

Results indicated no cancer recurrence during the period of AHCC consumption and no rise of tumor markers (CEA, CA 15-3); further, good quality of life was maintained. Further, there were no reports of adverse reactions thought to be caused by AHCC. In sum, we report a case in which a patient ingested AHCC for almost a decade after breast cancer surgery, maintaining good health status.

Abbreviations: AHCC: Active Hexose Correlated Compound, CEA: Carcinoembryonic Antigen, CA15-3: Carbohydrate antigen 153, QOL: Quality of Life

\section{Introduction}

Active Hexose Correlated Compound (AHCC) was developed in 1989 (Amino Up Chemical Co., Ltd.; Sapporo, Japan). This dietary supplement is obtained by the long-term liquid culturing of the mycelium of shiitake mushrooms (Lentinulla edodes). AHCC is widely used not only in Japan but also in Europe, America, Asia, and Oceania and has been adopted in medical facilities as a means of integrated medical care. The safety of AHCC has been confirmed by animal studies, single oral administration toxicity tests, single intraperitoneal administration toxicity tests, 4-month repeat toxicity tests, and mutagenicity tests. A human clinical trial conducted a safety test in healthy volunteers (phase I-like test), and no severe adverse reactions were observed, even with continuous intake of 3 times ( $9 \mathrm{~g} /$ day) the normally recommended amount for 2 weeks[1].

Lentinan, a type of $\beta$-glucan obtained from shiitake mushrooms, has been approved as a pharmaceutical product. It is reported that when used in combination with chemotherapy for cancer patients, there is a prolongation effect[2].

On the other hand, because AHCC is not medicine, cancer patients can obtain and use it at any time, including during or after treatment, based on individual preference.

Therefore, we conducted a follow-up survey from 2008 to 2016 for a patient who underwent breast cancer surgery and chemotherapy and had a long-term intake of AHCC ( $3 \mathrm{~g} /$ day) after treatment. We report on the transition of tumor markers (CEA, CA 15-3) and patient's quality of life.

\section{Case presentation}

Patient: Born in 1963, female, Irish, (53 years old as of 2017).

Diagnosis: Breast Cancer (2008); right breast, stage 2, triplenegative (i.e., negative for estrogen receptor, progesterone receptor, human epidermal growth factor receptor type 2), margin-negative, no metastasis (11 lymph nodes removed but all negative), tumor markers (CEA, CA15-3) above baseline.

Curative treatment: Surgery and chemotherapy (Paclitaxel, Adriamycin plus Cyclophosphamide) performed at Hokkaido University Hospital Department First Surgery (Sapporo, Japan) in April 2008. Subsequent blood tests and recurrence check were conducted at Hokkaido National Cancer Center (Sapporo, Japan). Patient returned from Japan to Northern Ireland in 2012. Blood from 2012 was tested at Antrim Area Hospital (Northern Ireland).

Cancer recurrence test: In April 2010, cells that seemed malignant as a result of a cytology test of body fluids were not found. In August 2010, CT examination revealed no recurrence or metastasis image of right breast cancer after surgery. No pathological lymph node

Correspondence to: Dr. Koji Wakame, Department of Pharmacology, Hokkaido Pharmaceutical University School of Pharmacy, 7-15-4-1 Maeda, Teine, Sapporo, Hokkaido, 006-8590 Japan, Tel: +81-676-8700, Fax:+81-676-8666, E-mail: wakame-k@hokuyakudai.ac.jp

key words: AHCC, QOL, integrated medical care, breast cancer, CEA, CA15-3

Received: February 17, 2017; Accepted: February 24, 2017; Published: February 27,2017 
Wakame K (2017) Long-term administration of Active Hexose Correlated Compound as a dietary supplement to a patient after breast cancer surgery and chemotherapy: A case report

enlargement was observed. No abnormality was found in the lungs. There were no metastatic images in the abdomen.

AHCC treatment: After surgery and chemotherapy, the patient began to consume $3 \mathrm{~g}$ AHCC per day, as per personal preference. The patient consumed AHCC regularly from September 2008 to March 2017, during which time she never ceased for more than 1 week. No severe adverse reactions considered to be caused by AHCC were reported.

Blood test and medical and lifestyle questionnaire: With the consent of the patient, we tracked the transition of tumor markers (CEA, CA 15-3) from September 2008 to January 2013. The patient completed a Medical and Lifestyle Questionnaire in January 2015.

Blood test data showed no abnormal values during the period of AHCC consumption (data not shown). For tumor markers, CEA (reference value $<5.0 \mathrm{ng} / \mathrm{mL}$ ), did not exceed the baseline level and showed a declining trend throughout the study period. CA 15-3 was slightly higher value than the reference value of $\leq 25.0 \mathrm{U} / \mathrm{mL}$ throughout the investigation period, but remained relatively stable (Figure 1).

Results of the Medical and Lifestyle Questionnaire indicated that the patient's lifestyle, health status, and quality of life (QOL) were good, and that little effect of surgery and chemotherapy for breast cancer remained (Table 1).

Other information: AHCC is a daily supplement in Japan and we have not gained ethical approval for this study because the patient adopted AHCC as part of her daily diet. The results of this test were submitted with patient written consent.

\section{Discussion}

In a previously reported double-blind, randomized human clinical trial in which AHCC ( $3 \mathrm{~g} /$ day) was administered for a period of one month and immune function in peripheral blood was measured. Results showed an increase in the total dendritic cell count and myeloid dendritic cell count, as well as an increase in the mixed lymphocyte culture response[3].

In another study of 222 patients with hepatocellular carcinoma who underwent hepatectomy, 113 patients received AHCC (3 g/ day) and patient prognoses were followed up retrospectively. Results revealed that the postoperative intake of AHCC resulted in significantly favorable outcomes in the recurrence and survival rate of hepatocellular cancer [4]. In a clinical, double blind trial of AHCC, 74 patients on watchful waiting with prostate cancer received AHCC ( $3 \mathrm{~g} /$ day) for 6 months. Results demonstrated good patient compliance, no serious adverse events, and increased QOL (alleviated anxiety)[5].

In a recent report, $\mathrm{AHCC}(3 \mathrm{~g} /$ day $)$ was administered to 24 patients who were receiving chemotherapy for colorectal cancer, pancreatic cancer, lung cancer, and ovarian cancer. Findings indicated increased QOL and decreased human herpesvirus 6 in saliva [6]. In another study, AHCC $(3 \mathrm{~g} / \mathrm{d})$ was administered to 18 breast cancer patients who were receiving chemotherapy (Anthracycline and Taxane) and results were compared to 23 patients who did not receive AHCC. Study results suggested that AHCC might reduce the severity of neutropenia induced by chemotherapy[7].

These reported benefits of AHCC may be a result of its modulatory action on the immune system, rather than its direct action on bacteria, viruses, or cancer cells. Production of interleukin- 6 and activation of natural killer (NK) cells, which are induced by a-glucan fractions from AHCC, are dependent on Toll-like receptors (TLR), and it is suggested that the receptors for AHCC may be TLR-2, TLR-4, or TLR6[8]. Studies in mouse models have reported that AHCC was effective in preventing influenza virus infections by increasing NK cell activity levels [9], and in preventing infections such as Candida albicans caused by decreased immune system due to cyclophosphamide (CY) administration, pseudomonad aeruginosa infections, and methicillinresistant staphylococcus aureus infections [10].

In summary, in animal and clinical studies it is reported that AHCC exerts biological defense mechanisms by activating the
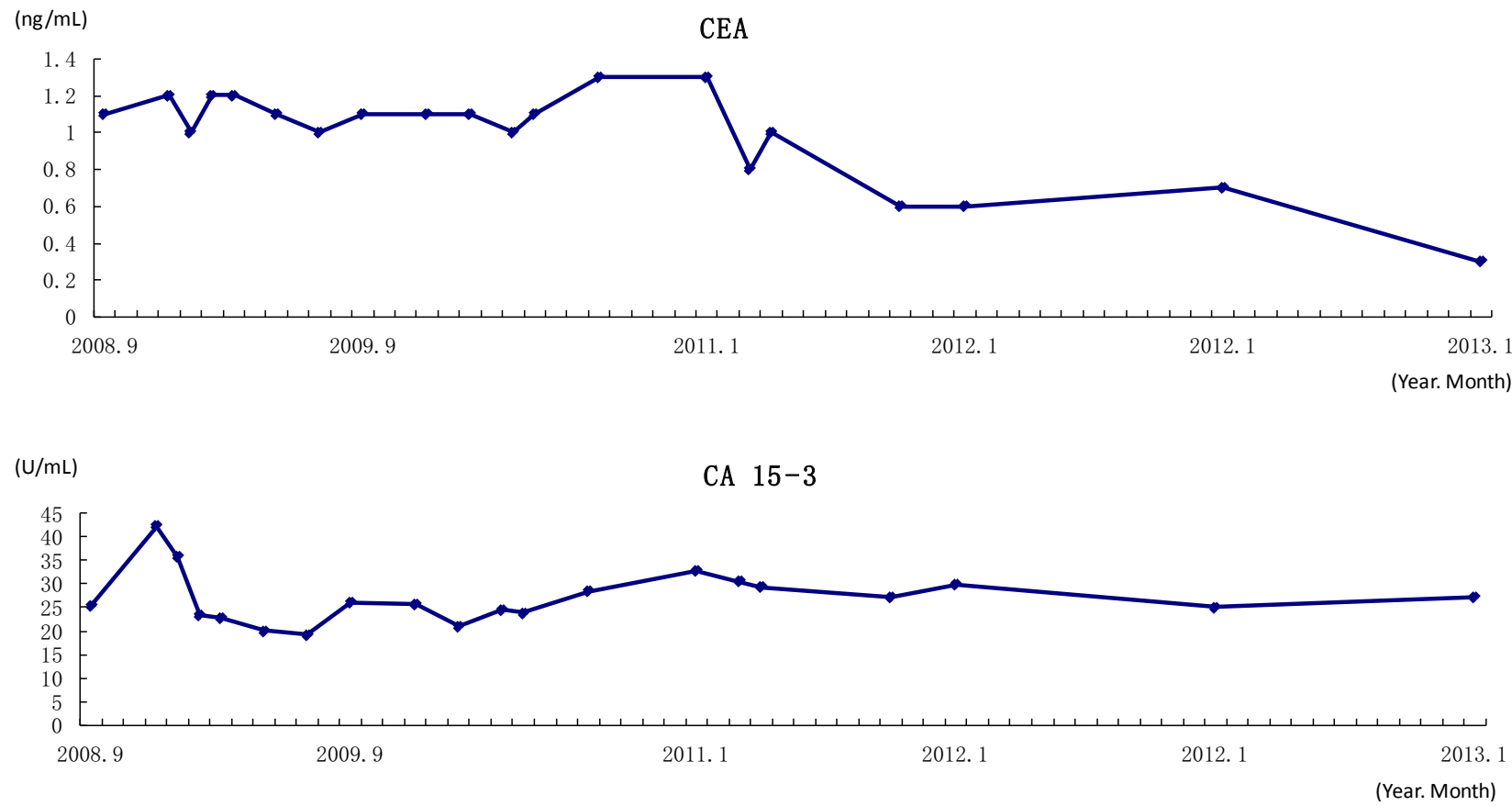

Figure 1. The transition of tumor markers (CEA, CA 15-3) from September 2008 to January 2013 
Wakame K (2017) Long-term administration of Active Hexose Correlated Compound as a dietary supplement to a patient after breast cancer surgery and chemotherapy: A case report

Table 1: Result of Medical and Lifestyle Questionnaire in January 2015

\section{Medical and Lifestyle Questionnaire}

\section{Questionnaire}

Ethnicity : Please indicate your cultural background, this is required to calculate some parameters within the profile.

Exercise : Please select from the list to indicate your normal exercise pattern.

Diet : Please select from the list to indicate your normal diet.

Smoking : Please select to indicate if you smoke.

Alcohol : Please indicate if you drink alcohol.

Travel History : Have you travelled outside of the United Kingdom in the last year? Please provide details.

Family Health : Have any 1st degree relatives (parent, brother, sister or child) aged $<60$ suffered a heart attack or angina?

Please give details of any medical conditions that members of your immediate family suffer or have suffered. Relative. Health History. Age if Living

Current Medication: Are you on blood pressure treatment?

Current Medication: Are you on cholesterol / triglyceride treatment?

Current Medication: Are you currently taking any other medications or supplements (e.g. vitamins) either prescribed or bought over the counter?

Medical History: Have you been diagnosed with diabetes?

Medical History: Have you been diagnosed with hypertension (high blood pressure)?

Medical History: Have you suffered a heart attack, angina, stroke or TIA?

Do you suffer from any other conditions?

Have you had any recent tests or investigations performed either by your GP or in hospital? Please provide details.

Do you have any head related symptoms? (for example, headaches, dizziness, dizziness when standing up, fainting or feeling faint)

Do you have any ear, nose or throat related symptoms? (for example, pain, congestion, hoarseness or hearing difficulty)

Do you have any eye or vision related symptoms? (for example, redness, pain, itching, blurred vision or loss of vision)

Do you have any neck or back symptoms? (for example, pain or stiffness)

Do you have any chest related symptoms? (for example, shortness of breath, pain, palpitations, wheezing or cough)

Do you have any abdominal symptoms? (for example, pain, tenderness, nausea, vomiting, bloating or heartburn)

Have you noticed any change in your bowel habits? (for example, more or less frequent, constipation, diarrhoea, flatulence, pain or bleeding)

Do you have any urinary symptoms? (for example, pain during urination, increased frequency or change in urine colour)

Do you have any upper limb related symptoms (shoulder, elbow or hand problems etc)? (for example, pain, stiffness, swelling, numbness or tingling)

Do you have any lower limb related symptoms (thigh, knee, foot or toe problems etc)? (for example, pain, stiffness, swelling, numbness or tingling)

Do you have any skin symptoms? (for example, rashes, itching, redness or swelling)

Do you suffer from difficulty sleeping, anxiety, irritability or depression?

Current Symptoms: Have you experienced any change in weight?

Current Symptoms: Have you experienced any change in appetite?

Current Symptoms: Are you on any contraceptive medication (e.g. pill, implant)?

Have you had your menopause?

Are your periods regular?

Do you have heavy menstrual periods? No First day of last menstrual period (if applicable)

Do you have bleeding between menstrual periods?

Have you experienced any hair loss?

Have you ever been pregnant? Further details (Optional)

Do you have any breast problems? (for example, pain, tenderness, lumps)

Do you have any symptoms or complaints not reported above? (for example, tiredness, thirst, high temperature, chills or sweating)

Answer
$(16 / 01 / 2015)$

White

Moderate exercise/sports 3-5 days/week Healthy

Non-Smoker No

America, Canada

No

Father Heart difficulties 77 yo

\begin{tabular}{|l} 
No \\
No \\
AHCC \\
No \\
No \\
No \\
No \\
No \\
\hline
\end{tabular}

Ultrasound scan Mammogram Bone scan - all test results clear.

\begin{tabular}{|c|}
\hline No \\
\hline No \\
\hline No \\
\hline No \\
\hline No \\
\hline No \\
\hline No \\
\hline No \\
\hline No \\
\hline No \\
\hline No \\
\hline No \\
\hline No \\
\hline No \\
\hline No \\
\hline Yes \\
\hline $14 / 01 / 2015$ \\
\hline Yes \\
\hline No \\
\hline Yes \\
\hline pregnancies \\
\hline No \\
\hline No \\
\hline
\end{tabular}

immune system. In particular, based on results of human clinical studies, it seems that AHCC intake can alleviate the adverse reactions of chemotherapy and maintain or improve patient QOL. Good patient compliance and maintaining QOL were also reported in the current case report, and it is particularly noteworthy that the patient experienced no serious adverse reactions during the long course of AHCC intake (i.e., 9 years). Similarly, because there was no increase in tumor markers and no recurrence of cancer, this case report provides physicians and other experts with valuable information regarding the long-term administration of AHCC after chemotherapy or other standard therapy.

To conclude, this case report suggests that long-term administration of AHCC as a dietary supplement after breast cancer surgery is safe and effective in maintaining health. In the future, further reports from physicians about cases of supplemental intake of AHCC are anticipated.

\section{References}

1. Spierings EL, Fujii H, Sun B and Walshe T (2007) A Phase I study of the safety of the nutritional supplement, active hexose correlated compound, AHCC, in healthy volunteers. J NutrSciVitaminol 53:536-539. [Crossref]

2. Ina K, Furuta R, Kataoka T, Kayukawa S, Yoshida T, et al. (2011) Lentinan prolonged survival in patients with gastric cancer receiving S-1-based chemotherapy. World $J$ ClinOncol 2: 339-343. [Crossref]

3. Terakawa N, Matsui Y, Satoi S, Yanagimoto H, Takahashi K, et al. (2007) Immunological effect of active hexose correlated compound (AHCC) in healthy volunteers: a doubleblind, placebo-controlled trial. Nutr Cancer 60: 643-651. [Crossref]

4. Matsui Y, Uhara J, Satoi S, Kaibori M, Yamada H, et al. (2002) Improved prognosis of postoperative hepatocellular carcinoma patients when treated with functional foods: a prospective cohort study. J Hepatol 37: 78-86. [Crossref]

5. Sumiyoshi Y, Hashine K, Kakehi Y, Yoshimura K, Satou T, et al. (2010) Dietary Administration of Mushroom Mycelium Extracts in Patients with Early Stage Prostate Cancers Managed Expectantly: A Phase II Study. Jpn J ClinOncol 40:967-972.[Crossref] 
Wakame K (2017) Long-term administration of Active Hexose Correlated Compound as a dietary supplement to a patient after breast cancer surgery and chemotherapy: A case report

6. Ito T, Urushima H, Sakaue M, Yukawa S, Honda H, et al. (2014) Reduction of Adverse Effects by a Mushroom Product, Active Hexose Correlated Compound (AHCC) in Patients With Advanced Cancer During Chemotherapy-The Significance of the Levels of HHV-6 DNA in Saliva as a Surrogate Biomarker During Chemotherapy. NutrCancer66: 377-382. [Crossref]

7. Hangai S, Iwase S, Kawaguchi T, Kogure Y, Miyaji T, et al. (2013) Effect of Active Hexose-Correlated Compound in Women Receiving Adjuvant Chemotherapy for Breast Cancer: A Retrospective Study. J AlternComplement Med 19: 905-910. [Crossref]
8. Mallet JF, Graham É, Ritz BW, Homma K, Matar C (2016) Active Hexose Correlated Compound (AHCC) promotes an intestinal immune response in BALB/c mice and in primary intestinal epithelial cell culture involving toll-like receptors TLR-2 and TLR-4. Eur J Nutr 55:139-146. [Crossref]

9. Nogusa S, Gerbino J, Ritz BW (2009) Low-dose supplementation with active hexose correlated compound improves the immune response to acute influenza infection in C57BL/6 mice. Nutr Res 29:139-143. [Crossref]

10. Aviles H, Belay T, Vance M, Sun B, Sonnenfeld G (2004) Active hexose correlated compound enhances the immune function of mice in the hindlimb-unloading model of spaceflight conditions. J ApplPhysiol 97: 1437-1444. [Crossref]

Copyright: (C2017 Wakame K. This is an open-access article distributed under the terms of the Creative Commons Attribution License, which permits unrestricted use, distribution, and reproduction in any medium, provided the original author and source are credited. 